\title{
Depth-Resolved Magnetization Dynamics Revealed by X-Ray Reflectometry Ferromagnetic Resonance
}

\author{
D. M. Burn $\odot,{ }^{1, *}$ S. L. Zhang, ${ }^{2,3, \uparrow}$ G. Q. Yu $\odot,{ }^{4}$ Y. Guang $\odot,{ }^{4}$ H. J. Chen $\odot,{ }^{5}$ X. P. Qiu, ${ }^{5}$ G. van der Laan $\odot,{ }^{1, \star}$ and T. Hesjedal $\odot^{6}$ \\ ${ }^{1}$ Diamond Light Source, Harwell Science and Innovation Campus, Didcot, Oxfordshire OX11 ODE, United Kingdom \\ ${ }^{2}$ School of Physical Science and Technology, ShanghaiTech University, Shanghai 200031, China \\ ${ }^{3}$ ShanghaiTech Laboratory for Topological Physics, ShanghaiTech University, Shanghai 200031, China \\ ${ }^{4}$ Beijing National Laboratory for Condensed Matter Physics, Institute of Physics, Chinese Academy of Sciences, Beijing 100190, China \\ ${ }^{5}$ Shanghai Key Laboratory of Special Artificial Microstructure Materials and School of Physics Science and Engineering, \\ Tongji University, Shanghai 200092, China \\ ${ }^{6}$ Department of Physics, Clarendon Laboratory, University of Oxford, Oxford OX1 3PU, United Kingdom
}

(Received 8 July 2020; revised 29 July 2020; accepted 13 August 2020; published 24 September 2020)

\begin{abstract}
Magnetic multilayers offer diverse opportunities for the development of ultrafast functional devices through advanced interface and layer engineering. Nevertheless, a method for determining their dynamic properties as a function of depth throughout such stacks has remained elusive. By probing the ferromagnetic resonance modes with element-selective soft x-ray resonant reflectivity, we gain access to the magnetization dynamics as a function of depth. Most notably, using reflectometry ferromagnetic resonance, we find a phase lag between the coupled ferromagnetic layers in $[\mathrm{CoFeB} / \mathrm{MgO} / \mathrm{Ta}]_{4}$ multilayers that is invisible to other techniques. The use of reflectometry ferromagnetic resonance enables the time-resolved and depth-resolved probing of the complex magnetization dynamics of a wide range of functional magnetic heterostructures with absorption edges in the soft x-ray wavelength regime.
\end{abstract}

DOI: 10.1103/PhysRevLett.125.137201

A detailed understanding of the contributions from the constituent entities in composite materials systems is key to achieving advanced functionalities. This is especially true for magnetic materials in which intricate coupling phenomena can be exploited on all length scales-from microscopic superexchange to macroscopic interactions [1]. Magnetic multilayers [2] are a prime example of advanced materials in which functionalities can be precisely engineered through control of the layer properties, the coupling across the interfaces and between layers. These techniques have enabled the massive downscaling of magnetic memory [3] and contributed to exciting developments in fields such as skyrmions in topological magnetic materials [4,5], synthetic antiferromagnets [6], and spintronic devices [7]. Nevertheless, most established magnetic characterization techniques aim at the macroscopic properties, and are only sensitive to the total magnetization of a material, and unable to discriminate between contributions from different atoms or layers. With increasing demand for materials for high frequency applications, techniques for

Published by the American Physical Society under the terms of the Creative Commons Attribution 4.0 International license. Further distribution of this work must maintain attribution to the author(s) and the published article's title, journal citation, and DOI. probing both the depth-resolved and time-resolved magnetization dynamics are required.

The use of x-ray reflectivity has become commonplace for characterizing the depth-dependent structure of layered materials [8]. This nondestructive technique probes x-ray interference effects between layers, and, at resonance with an element-specific absorption energy, additional insight into the magnetic structure is obtained [9-11]. Here, the element-specific magnetic sensitivity originates from $\mathrm{X}$-ray linear and circular magnetic dichroism (XMLD and XMCD) $[12,13]$. These synchrotron-based spectroscopies have had a large impact on the microscopic understanding of magnetic interactions and offer applications in magnetic materials studies across a wide range of scientific disciplines [14].

Ferromagnetic resonance (FMR), commonly used to probe magnetization dynamics in the frequency domain, can be combined with XMCD in a technique called X-ray detected ferromagnetic resonance (XFMR) $[15,16]$. While FMR only probes the weighted average of the sample response, XFMR can resolve the layer-specific magnetization dynamics in few-layer systems if the layers differ in chemical contrast. Taking advantage of the pulsed time structure of synchrotron radiation, XFMR has been exploited to extract the amplitude and relative phase of precession in individual magnetic layers of spin valve structures [17-19] and even on magnetic diffraction peaks [20,21]. However, while XFMR can probe few-layer systems, the depth 
dependence to the magnetization dynamics from generalized composite materials remains elusive.

In this Letter, we introduce reflectometry FMR (RFMR), a technique in which FMR is combined with $x$-ray resonant reflectivity to reveal the depth-dependent dynamics within magnetic heterostructures. RFMR builds on interference effects that arise due to reflections from the layered structure using a length scale that matches well to the soft $\mathrm{x}$-ray wavelength. The reflectivity therefore provides a unique view into the dynamic coupling within layered systems. Advantageously, reflectivity is not tied to fulfilling a diffraction condition, thereby allowing for a very large variety of materials systems to be investigated. Our $[\mathrm{CoFeB} / \mathrm{MgO} / \mathrm{Ta}]_{4}$ multilayers host a variety of topological magnetic phases [22]. Here, we reveal the depth dependence to the dynamics in the field-polarized phase exhibiting a conical precession with a phase lag between the magnetization precession in adjacent layers. Understanding of the depth-dependent dynamics in this system is important to the development of heterostructurebased functional magnetic devices in general.

The soft x-ray reflectivity was measured in the invacuum scattering chamber RASOR on beamline I10 at the Diamond Light Source. Left-circularly polarized x-rays with their energy tuned to either the Fe $L_{3}$ absorption edge at $707.7 \mathrm{eV}$ or off-edge at $700 \mathrm{eV}$ were incident upon the sample under variable angles $\theta$ with a $\sim 100 \mu \mathrm{m}$ spot size. The intensity of the reflected beam is detected by a photodiode in a $\theta-2 \theta$ geometry, as illustrated in the insert in Fig. 1(a).

First of all, the static reflectivity was measured as a function of the wave vector $Q_{z}$ with $H_{\text {Bias }}=7 \mathrm{mT}$ applied out of plane. Figure 1(a) shows a steep falloff in the reflected intensity, coupled with Kiessig fringes expected from interference effects between the layers [23]. These features contain information on the depth dependence of the charge density across the multilayer stack. Furthermore, modifications to this reflectivity at the $\mathrm{Fe} L_{3}$ edge result from resonant absorption effects where magnetic ordering contributes to the contrast and can be used to reveal the depth-dependent magnetization orientation throughout the multilayer.

The black lines in Fig. 1(a) show the simulated reflectivity from a model fit to the data [24,25]. This model is based on the depth dependence to the scattering length density (SLD) displayed in Fig. 1(b). The four repeat units in the multilayer stack are distinctly visible due to the layer composition differences in the SLD. For $\mathrm{MgO}$ and $\mathrm{Ta}$, there is little variation in the SLD between on- and offresonance measurements. However, the CoFeB layers show a dramatic difference due the presence of Fe. This modification to the SLD depends on the relative orientation of the magnetization. It results in a magnetic reflectivity contrast as a function of scattering vector that is directly connected to the magnetization in the different layers.
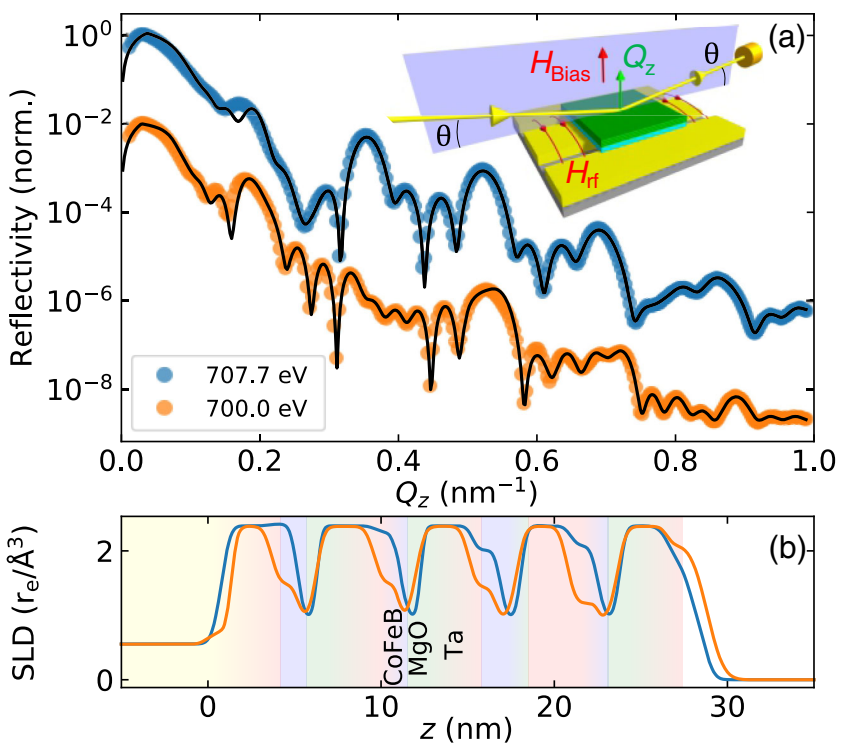

FIG. 1. (a) Specular x-ray reflectivity of $[\mathrm{CoFeB} / \mathrm{MgO} / \mathrm{Ta}]_{4}$ multilayer as a function of wave vector $Q_{z}$, measured with circularly polarized x-rays at 707.7 and $700 \mathrm{eV}$ (i.e., on and off resonance at the $\mathrm{Fe} L_{3}$ edge, respectively) in an out-of-plane bias field $H_{\text {Bias }}=7 \mathrm{mT}$. Black lines represent fits to the data based on the model illustrated in (b), where the SLD is plotted as a function of depth $z$. The inset in (a) shows a schematic of the experimental setup with the sample (green) mounted on a coplanar waveguide (yellow) in the $\theta-2 \theta$ scattering configuration with the scattering vector $Q_{z}$. The field $H_{\text {Bias }}$ is out of plane, and the rf field $H_{\mathrm{rf}}$ is in plane near the sample surface and perpendicular to the scattering plane (purple).

In the dynamic regime, the magnetization in each $\mathrm{CoFeB}$ layer is driven into precession. This oscillatory magnetization results in time-dependent changes in the reflected intensity due to the rf field that occur in addition to a static reflectivity contribution. We define the dynamic reflectivity as the time-dependent change that is sinusoidal in nature and that can be expressed in terms of its amplitude and phase. The dynamic reflectivity is probed using the stroboscopic XFMR method where an rf magnetic field, $H_{\mathrm{rf}}$, generated by the coplanar waveguide beneath the sample was used to drive the magnetization dynamics in the sample [see inset to Fig. 1(a)]. The rf field was phase-locked to the fourth harmonic of the $\sim 500 \mathrm{MHz}$ synchrotron master clock at $2 \mathrm{GHz}$, providing synchronization between the magnetization dynamics and the photon pulses arriving at the sample [26]. The time dependence of the reflectivity during precession was mapped out as a function of time delay between the rf pump and x-ray probe. The signal-to-noise ratio of the signal was enhanced through lock-in techniques, where a $180^{\circ}$ phase modulation at $2.3 \mathrm{kHz}$ was applied to the $\mathrm{rf}$ drive signal.

Figure 2 shows an example of the dynamic contribution to the specular reflectivity as a function of time delay. 


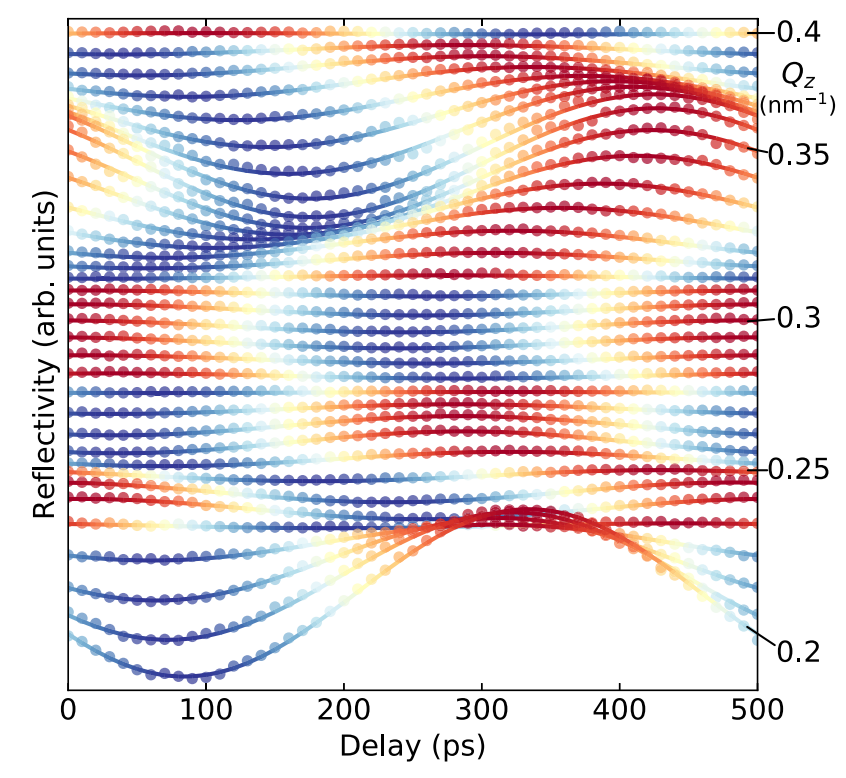

FIG. 2. Waterfall plot showing the dynamic contribution to the reflectivity for $[\mathrm{CoFeB} / \mathrm{MgO} / \mathrm{Ta}]_{4}$ multilayer as a function of pump-probe time delay. The measurements were carried out with left-circularly polarized x-rays at resonance $\left(707.7 \mathrm{eV}, \mathrm{Fe} L_{3}\right)$ and in an out-of-plane field of $29 \mathrm{mT}$. Various delay curves are shown for $Q_{z}$, ranging between 0.2 and $0.4 \mathrm{~nm}^{-1}$. The color scale represents the normalized intensity (shifted vertically for clarity) for each delay scan, highlighting the sinusoidal dependence and the shift in phase as $Q_{z}$ is varied when the intensity is small. The lines represent sinusoidal fits to the data points.

These measurements were performed on the multilayer sample in an out-of-plane saturating field of $H_{\text {Bias }}=$ $29 \mathrm{mT}$ and are shown for a range of $Q_{z}$ values. The intensity follows a sinusoidal dependence on the pumpprobe delay with a $500 \mathrm{ps}$ period, originating from the $2 \mathrm{GHz}$ rf excitation. The dynamic signal changes as a function of $Q_{z}$ in both amplitude and phase. The amplitude variations are most pronounced for the low $Q_{z}$ regime around $0.2 \mathrm{~nm}^{-1}$, where a large static reflectivity is measured. The phase of the dynamic signal shows a complex behavior as a function of $Q_{z}$, which is illustrated by the displacements and is further highlighted by the color scale applied to the data points in Fig. 2. This behavior includes slow, smooth phase variations as a function of $Q_{z}$, in addition to abrupt changes where the phase shifts by $180^{\circ}$.

Further insight into the dynamic reflectivity is extracted from sinusoidal fits to the delay scans. These fits are parameterized by an amplitude and phase that are shown in Fig. 3 as a function of $Q_{z}$. The behavior of the dynamic reflectivity is similar to the static signal but at a reduced intensity and with several additional sharp minima. The blue-shaded regions indicate the $Q_{z}$ range at which the phase shifts by $180^{\circ}$; these phase shifts have been subtracted to highlight the underlying phase variations. For comparison, the untreated data (before subtracting the $180^{\circ}$

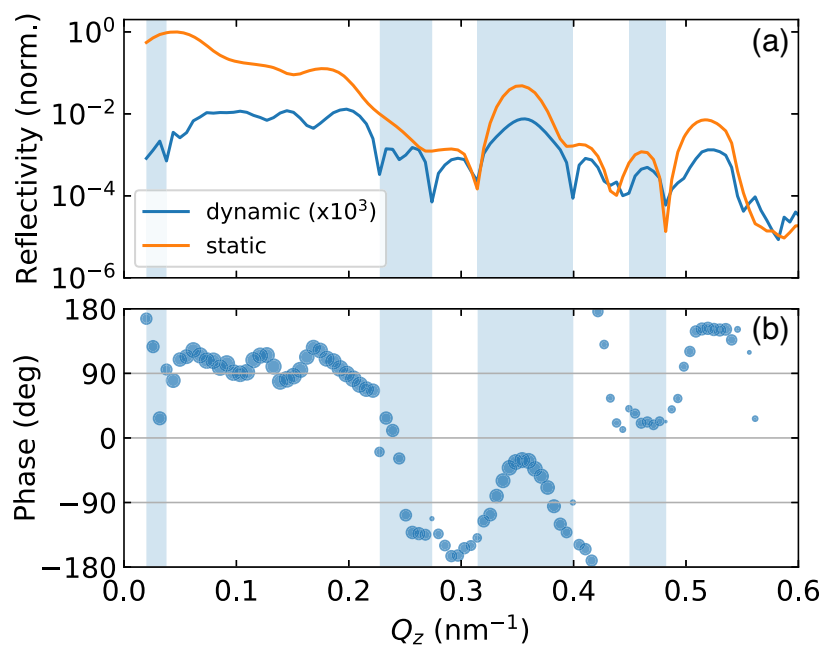

FIG. 3. (a) Static and dynamic reflectivity, and (b) phase of the dynamic reflectivity as a function of $Q_{z}$ for $[\mathrm{CoFeB} / \mathrm{MgO} / \mathrm{Ta}]_{4}$ multilayer. Measurements were performed with left-circularly polarized x-rays at resonance $\left(707.7 \mathrm{eV}, \mathrm{Fe} L_{3}\right)$ using rf excitation at $2 \mathrm{GHz}$. The phase point size is scaled by the dynamic signal amplitude and the blue-shaded regions indicate where the $180^{\circ}$ phase shifts have been subtracted to reveal the otherwise smooth phase variation (see Fig. S1 in the Supplemental Material [27] for more details).

phase shifts) is shown in Fig. S1 in the Supplemental Material [27].

The $180^{\circ}$ phase shifts are reminiscent of previous reports on XFMR [28]. Here, the dynamic signal, which is measured for fixed circular polarization, has a positive (negative) sign when the magnetization precession is in phase (antiphase) with the rf. When the XMCD spectrum changes sign as a function of photon energy, e.g., by going from the $L_{3}$ to the $L_{2}$ absorption edge, the XFMR signal reverses sign as well. This leads to an apparent $180^{\circ}$ phase shift. The same occurs in RFMR when the circular dichroism in reflectivity changes sign as a function of $Q_{z}$. This has been further elaborated in Fig. S2 in the Supplemental Material [27]. It is interesting to note that the $180^{\circ}$ phase shifts coincide with the sharp minima in the dynamic reflectivity (see Fig. 3). This is not accidental. However, it is also not a hard and fast rule that pronounced minima are necessarily related to $180^{\circ}$ phase shifts.

Much of the dynamic reflectivity behavior can be reproduced by extending the static model in Fig. 1 to the dynamic regime by simulating the reflectivity from multiple subsequent snapshots while a conical precession is applied to the nominally out-of-plane moments in each magnetic layer within the multilayer. Following the same analysis as for the experimental results, both the static and dynamic contributions to the reflectivity are extracted from the model and are shown in Fig. 4(a),(b) as a function of $Q_{z}$. In this example, the magnetic moments in all four layers precess in phase, mapping a cone with an opening angle of $\alpha=1^{\circ}$, as shown in the inset of Fig. 4(b). 

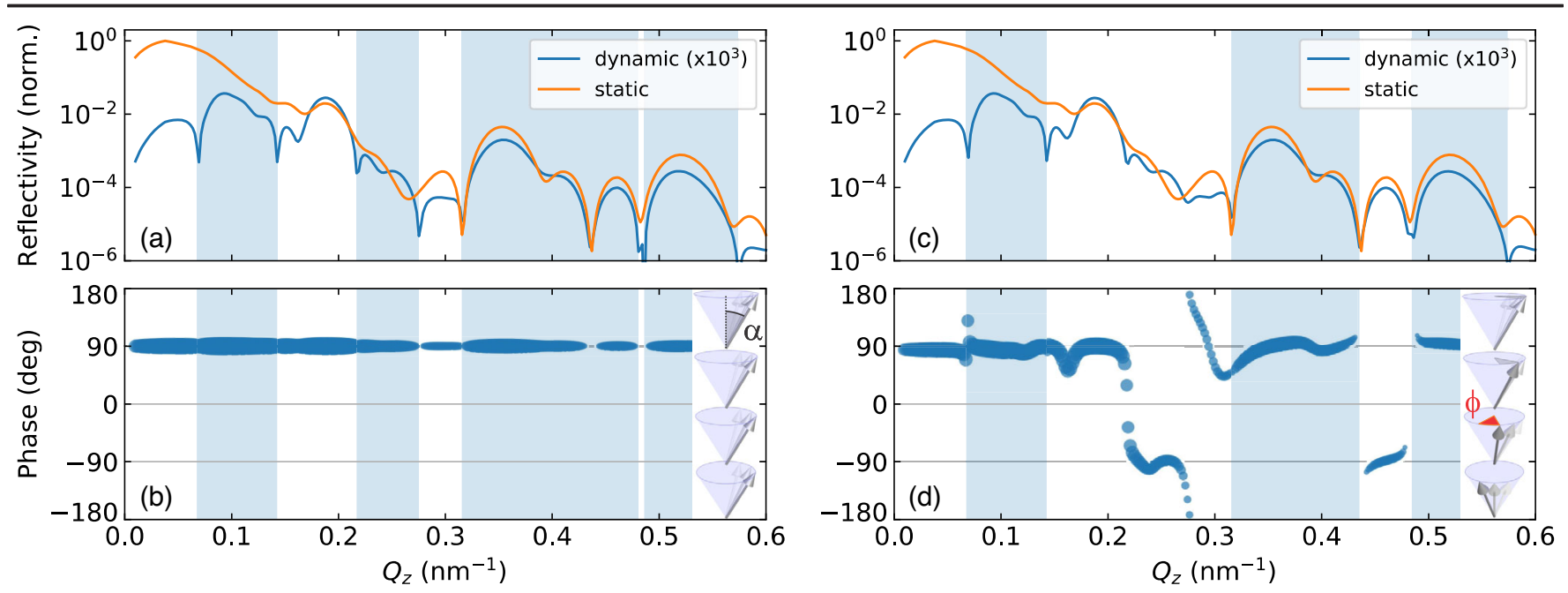

FIG. 4. (a),(c) Static and dynamic contributions to the reflected intensity, and (b),(d) the phase of the dynamic reflectivity simulated from a multilayer model. The magnetization in all layers precesses with a $\alpha=1^{\circ}$ cone angle about the surface normal direction. This precession is either in phase, (a),(b), or includes a $\phi=5^{\circ}$ phase lag between adjacent layers, (c),(d), as illustrated in the insets to the bottom panels. The phase point size is scaled by the dynamic signal amplitude and the $180^{\circ}$ phase shifts have been subtracted in the blueshaded regions, revealing either (b) a negligible or (d) a significant residual phase dependence on $Q_{z}$.

The general shape of the dynamic signal follows that of the static signal at a reduced intensity. Furthermore, the $180^{\circ}$ phase changes, which are subtracted in the blue-shaded regions, coincide with sharp minima in the dynamic reflectivity. This model, however, shows no remaining variations in phase after the subtraction of the $180^{\circ}$ phase shifts.

For the modeling presented here, a precessional cone angle of $\alpha=1^{\circ}$ was used. The angle $\alpha$ scales the magnitude of the dynamic reflectivity, but it has no influence on the shape of the reflectivity signal or the dynamic phase. Furthermore, the inclusion of the phase lag does not change the static reflectivity, as can be seen by comparing Fig. 4(a) and 4(c) as it results from the time-averaged precessional dynamics. The dynamic intensity does, however, show some small variations, particularly in the $Q_{z}$ region from 0.2 to $0.3 \mathrm{~nm}^{-1}$ where changes in phase are most significant.

Next, we discuss adding a phase lag of $\phi=5^{\circ}$ between the precession in adjacent magnetic layers as shown in the inset of Fig. 4(d). Figure 4(c), (d) show both the static and dynamic contributions to the reflectivity resulting from this phase lag, where significant variations in the RFMR phase now occur, in addition to the $180^{\circ}$ phase shifts that have been subtracted in the blue-shaded regions. These residual phase variations show significant similarities with those observed in the experimental results for the multilayer sample. For comparison, dynamic reflectivity measurements on a single CoTb thin film slab show the abrupt $180^{\circ}$ phase shifts but no additional variation in the phase (see Fig. S3 in Supplemental Material [27]). This single slab behavior is well described by the model, which does not include a phase lag in Fig. 4(a), (b).
This leads us to conclude that the discontinuous structure in the multilayer alters the exchange coupling between layers, allowing for variations in the orientation of the magnetization in the dynamic regime. The multilayer is then able to adopt a depth-dependent dynamic structure where the inclusion of a phase lag between the layers is necessary to explain the experimental data. In fact, the $[\mathrm{CoFeB} / \mathrm{MgO} / \mathrm{Ta}]_{4}$ multilayer investigated here are known to host noncollinear magnetic structures stabilized by the Dzyaloshinskii-Moriya interaction, which arises at interfaces between a magnetic layer and a heavy metal with large spinorbit coupling [22]. The combination of the DzyaloshinskiiMoriya interaction and dipole interaction leads to the formation of complex three-dimensional noncollinear structures, where, e.g., the domain wall configuration continuously evolves throughout the stack, going from Néel-type to Bloch-type and back again to the inverse Néel-type [29]. Such complex three-dimensional magnetic structures lead to novel dynamics, and RFMR is the ideal tool to investigate the spin dynamics in a layer-by-layer fashion.

In conclusion, we have demonstrated that our RFMR technique allows for the characterization of the magnetization dynamics in a multilayer structure as a function of depth. As can be seen by comparing the experimental results with modeling of the dynamic behavior, excellent agreement has been achieved, thereby revealing the depthdependent magnetization dynamics. Here, the magnetization in all layers in our multilayered model precess about a nominal static state when excited by an rf field. We showed that the inclusion of a small but significant phase lag between the various layers is necessary to explain the observed change in phase of the dynamic signal. This is in contrast to the depth dependence of a single slab of 
magnetic thin film material in which coherent precession of the magnetization occurs as a function of depth. The nonmagnetic layers within the multilayer provide gaps over which exchange coupling is weak, allowing the independent dynamic orientation of the magnetization within each layer. With RFMR, the dynamics from different layers containing the same element can be explored, and there is the potential to study the dynamics of interfacial layers and proximity effects in a novel way. RFMR has the unique potential to explore complex thin film and multilayer materials for future magnetic memory and processing device applications.

The RFMR experiments were carried out in the RASOR scattering chamber on beamline I10 at the Diamond Light Source, UK, under proposal MM23895. We are indebted to Mark Sussmuth (I10) for his tireless technical support. Financial support from the Engineering and Physical Sciences Research Council (UK) under Grant No. EP/ N032128/1 is gratefully acknowledged. G. Y. acknowledges financial support from the National Natural Science Foundation of China (Grant No. 11874409) and the Beijing Natural Science Foundation (Grant No. Z190009). The authors appreciate the support from the Analytical Instrumentation Center (SPSTAIC10112914), School of Physical Sciences and Technology, ShanghaiTech University. S. L.Z. acknowledges the starting grant from ShanghaiTech University and the Eastern Scholar Scheme.

*Corresponding author.

david.burn@diamond.ac.uk

${ }^{\dagger}$ Corresponding author.

zhangshl1@shanghaitech.edu.cn

\#Corresponding author.

Gerrit.vanderLaan@diamond.ac.uk

[1] J. M. D. Coey, Magnetism and Magnetic Materials (Cambridge University Press, Cambridge, England, 2010).

[2] R. E. Camley and R. L. Stamps, J. Phys. Condens. Matter 5, 3727 (1993).

[3] S. Parkin, X. Jiang, C. Kaiser, A. Panchula, K. Roche, and M. Samant, Proc. IEEE 91, 661 (2003).

[4] A. Fert, N. Reyren, and V. Cros, Nat. Rev. Mater. 2, 17031 (2017).

[5] W. Jiang, G. Chen, K. Liu, J. Zang, and S. G. E. te Velthuis, and A. Hoffmann, Phys. Rep. 704, 1 (2017).

[6] R. A. Duine, K. J. Lee, S. S. P. Parkin, and M. D. Stiles, Nat. Phys. 14, 217 (2018).

[7] A. Brataas, A. Kent, and H. Ohno, Nat. Mater. 11, 372 (2012).

[8] K. N. Stoev and K. Sakurai, Spectrochim. Acta B Atom. Spectros. 54, 41 (1999).

[9] J. M. Tonnerre, L. Sève, A. Barbara-Dechelette, F. Bartolomé, D. Raoux, V. Chakarian, C. C. Kao, H. Fischer, S. Andrieu, and O. Fruchart, J. Appl. Phys. 83, 6293 (1998).
[10] S. Macke and E. Goering, J. Phys. Condens. Matter 26, 363201 (2014).

[11] C. Kao, J. B. Hastings, E. D. Johnson, D. P. Siddons, G. C. Smith, and G. A. Prinz, Phys. Rev. Lett. 65, 373 (1990).

[12] G. van der Laan, B. T. Thole, G. A. Sawatzky, J. B. Goedkoop, J. C. Fuggle, J. M. Esteva, R. Karnatak, J. P. Remeika, and H. A. Dabkowska, Phys. Rev. B 34, 6529 (1986).

[13] G. Schütz, W. Wagner, W. Wilhelm, P. Kienle, R. Zeller, R. Frahm, and G. Materlik, Phys. Rev. Lett. 58, 737 (1987).

[14] G. van der Laan and A. I. Figueroa, Coord. Chem. Rev. 277-278, 95 (2014).

[15] J. Goulon, A. Rogalev, F. Wilhelm, N. Jaouen, C. GoulonGinet, G. Goujon, J. Ben Youssef, and M. V. Indenbom, JETP Lett. 82, 696 (2005).

[16] G. van der Laan, J. Electron Spectrosc. Relat. Phenom. 220, 137 (2017).

[17] A. A. Baker, A. I. Figueroa, C. J. Love, S. A. Cavill, T. Hesjedal, and G. van der Laan, Phys. Rev. Lett. 116, 047201 (2016).

[18] J. Li, L. R. Shelford, P. Shafer, A. Tan, J. X. Deng, P. S. Keatley, C. Hwang, E. Arenholz, G. van der Laan, R. J. Hicken, and Z. Q. Qiu, Phys. Rev. Lett. 117, 076602 (2016).

[19] M. Dabrowski, T. Nakano, D. M. Burn, A. Frisk, D. G. Newman, C. Klewe, Q. Li, M. Yang, P. Shafer, E. Arenholz, T. Hesjedal, G. van der Laan, Z. Q. Qiu, and R. J. Hicken, Phys. Rev. Lett. 124, 217201 (2020).

[20] D. M. Burn, S. Zhang, K. Zhai, Y. Chai, Y. Sun, G. van der Laan, and T. Hesjedal, Nano Lett. 20, 345 (2020).

[21] S. Pöllath, A. Aqeel, A. Bauer, C. Lou, H. Ryll, F. Radu, C. Pfleiderer, G. Woltersdorf, and C. H. Back, Phys. Rev. Lett. 123, 167201 (2019).

[22] W. Li et al., Adv. Mater. 31, 1807683 (2019).

[23] J. Als-Nielsen and D. McMorrow, Elements of Modern X-Ray Physics (Wiley, New York, 2001).

[24] L. G. Parratt, Phys. Rev. 95, 359 (1954).

[25] M. Björck and G. Andersson, J. Appl. Crystallogr. 40, 1174 (2007).

[26] G. B. G. Stenning, L. R. Shelford, S. A. Cavill, F. Hoffmann, M. Haertinger, T. Hesjedal, G. Woltersdorf, G. J. Bowden, S. A. Gregory, C. H. Back, P. A. J. de Groot, and G. van der Laan, New J. Phys. 17, 013019 (2015).

[27] See Supplemental Material at http://link.aps.org/supplemental/ 10.1103/PhysRevLett.125.137201 for the as-measured dynamic signal, the identification of phase changes based on the static reflectivity asymmetry ratio, the dynamic reflectivity of $\mathrm{CoTb}$, and details on the sample growth.

[28] M. K. Marcham, P. S. Keatley, A. Neudert, R. J. Hicken, S. A. Cavill, L. R. Shelford, G. van der Laan, N. D. Telling, J. R. Childress, J. A. Katine, P. Shafer, and E. Arenholz, J. Appl. Phys. 109, 07D353 (2011).

[29] W. Legrand, J.-Y. Chauleau, D. Maccariello, N. Reyren, S. Collin, K. Bouzehouane, N. Jaouen, V. Cros, and A. Fert, Sci. Adv. 4, eaat0415 (2018). 